\title{
Towards a single-chip, implantable RFID system: is a single-cell radio possible?
}

\author{
Peter Burke • Christopher Rutherglen
}

Published online: 24 January 2009

(C) The Author(s) 2009. This article is published with open access at Springerlink.com

\begin{abstract}
We present an overview of progress towards single-chip RFID solutions. To date heterogeneous integration has been appropriate for non-biological systems. However, for in-vivo sensors and even drug delivery systems, a small form factor is required. We discuss fundamental limits on the size of the form factor, the effect of the antenna, and propose a unified single-chip RFID solution appropriate for a broad range of biomedical in-vivo device applications, both current and future. Fundamental issues regarding the possibility of single cell RF radios to interface with biological function are discussed.
\end{abstract}

\section{Keywords Sensor RFID}

\section{Introduction}

When James Clerk Maxwell discovered the displacement current, hence completing Maxwell's equations, in 1865, the stage was set for the wireless transmission of information. It another 75 years for the semiconductor industry to be born and mature into the field it is today. The modicum anytime anywhere access to information is now taken for granted. Today, every student in every science and engineering field is taught the fundamental basis of electromagnetics and wireless propagation of signals and information.

The next 100 years will see a similar revolution in the integration of information technology and biotechnology,

P. Burke $(\bowtie) \cdot$ C. Rutherglen

Department of Electrical Engineering and Computer Science,

University of California, Irvine,

Irvine, CA 92697, USA

e-mail: pburke@uci.edu also called biomedical engineering. The field of telemedicine (e.g. scanned patient records, email or virtual office visits, etc.) has access to wireless technology and is beginning to deploy and utilize the vast, low cost information processing capacity. In this paper, we wish to address a more general issue, that of interfacing the physical world with the biological world.

For interrogation of biological systems, one is generally interested in a chemical or physical quantity. For a chemical quantity, typical assays determine the presence or concentration of a protein, antibody, or small molecule anylate, the presence or concentration of a particular DNA or RNA, or even more subtle quantities such as the phosphorylation state of an enzyme.

In general, these biomedically relevant physical quantities are sensed and turned into a measurable optical or electronic signal. Because of the broad availability of low cost high performance electronics, the development of electronic technologies will be more of concern to us here. The use of electronic interrogation of biological function can be integrated into a Si CMOS chip at potentially low cost. However, there is an important issue of how to interface the CMOS chip to the outside world, and this is where RFID comes in.

The use of RFID for identification and sensing has been demonstrated in many applications. In general the acronym is used to refer only to identifying the presence of an RFID system. However, in this paper we wish to extend this definition and propose a more general RFID system that can both identify its presence and also interrogate its local chemical environment. Thus, in this work, RFID is used not just to refer to identification, but rather to measure and possibly effect biological function.

Figure 1 below poses the central question of this paper: Is a single-cell radio possible? Such a system would be able 
Single cell radio:

Science fiction or golden opportunity?

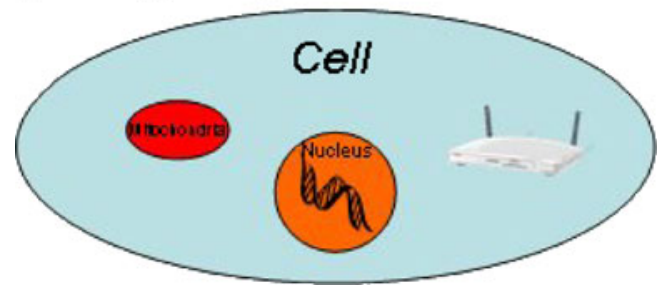

Fig. 1 Single cell radio concept

to interrogate and possibly affect biological function inside a single, living cell. This paper is not to be considered a comprehensive or complete review, but rather a discussion of some fundamental issues in scaling down RFID to the single cell level, and a presentation of future research directions.

\section{RFID state of the art}

The field of RFID in general is a complex field, with many applications in industry, medicine, and commerce (Finkenzeller 2003; Ahson and Ilyas 2008). Overall size reduction is not the primary goal in most applications; rather the cost is the most important factor. The development of single chip RFID solutions is not then the primary goal of industry to date. However, in this paper we argue that it could lead to lower cost, and especially smaller size, for biomedical implants. In addition, it seems the most appropriate technology vector for implantable biomedical microdevices. We next review current efforts in this direction.

\subsection{Small chips}

Hitachi(Usami et al. 2003, 2007) has developed technology for progressively smaller die sizes for RFID tags. The latest size is $50 \times 50 \times 5 \mu \mathrm{m}$, and shown as an example in Fig. 2 (compiled from Ref. (Usami et al. 2007)) below. This demonstrates the feasibility of small (microscopic) chips for RFID, however the antenna was external and adds significantly to the system size, discussed next.

\subsection{Big antennas}

Although the Hitachi work has demonstrated very small die sizes for the memory, the antenna must be external, and is typically $\mathrm{cm}$ or so in size. This is generally achieved via an off-chip antenna. However, recent research in on-chip antennas(Bouvier et al. 1997; Abrial et al. 2001; O et al.
2005; Shamin et al. 2005, 2008; Guo et al. 2006) has demonstrated the ability to fabricate smaller RF antennas on the same chip as the signal processing components. Using either $\mathrm{GHz}$ near-field antenna or $\mathrm{MHz}$ inductively coupled coils, researchers have shown of order $1 \mathrm{~mW}$ of available DC power on chip (from the RF field) in an area of order $1 \mathrm{~mm}^{2}$.

In Fig. 3 (compiled from Refs. (Guo et al. 2006; Usami et al. 2007)), the OCA operates at $2.45 \mathrm{GHz}$. The on-chip circuitry uses the energy from the incoming RF field to power itself, so that no battery is needed. In this case, $1 \mathrm{~mW}$ is available to power the on-chip circuitry. The antenna size is roughly a few $\mathrm{mm}$ by a few $\mathrm{mm}$. As Fig. 3 shows, the antenna is still much larger than the active circuitry of the Hitachi microchip, inserted to scale for reference. Thus, while a major advance in integration and size reduction (compared to the $\mathrm{cm}$ scale external antennas typically used), there is still vast room for improvement in miniaturization of this RFID device.

\subsection{Biomedical/commercial efforts}

It is one thing to demonstrate a lab prototype, but another to demonstrate and certify and in-vivo system. Several commercialization efforts towards this end are underway at various stages of maturity already. At least three companies have developed implantable RFID sensors (SMS, BioRasis, and ISSYS). SMS and BioRasis have targeted glucose sensors. ISSYS has targeted MEMs based pressure sensors. While detailed designs are not published,

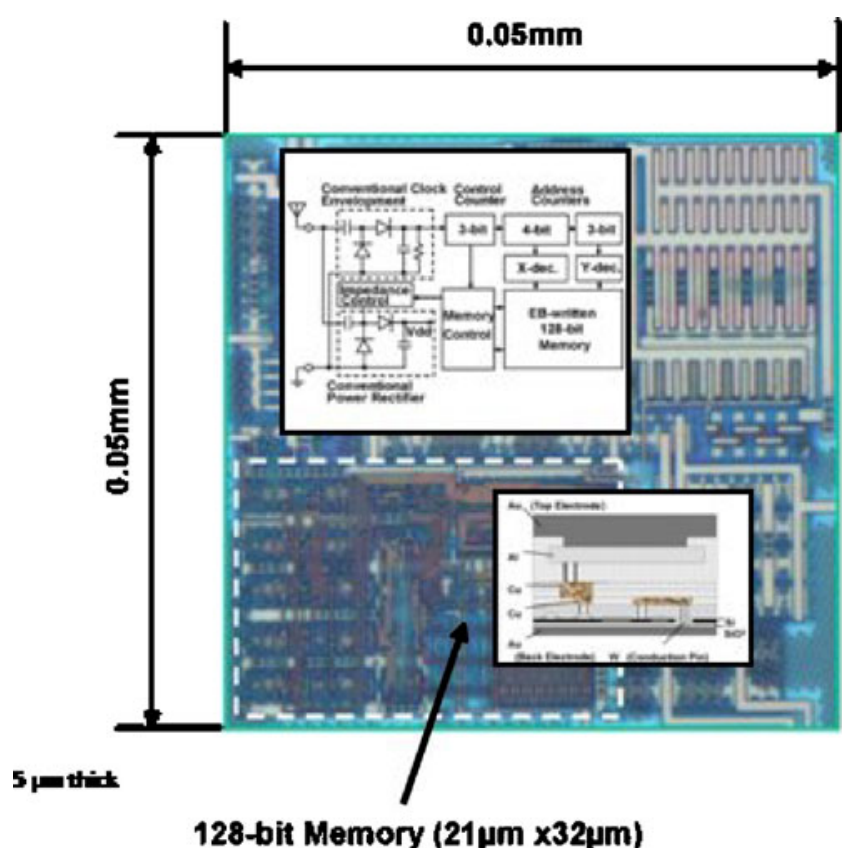

Fig. 2 Hitachi micro-chip. (C) 2006 IEEE 
Fig. 3 On-chip-antenna and

Hitachi micro-chip to scale.

(C) 2007 IEEE

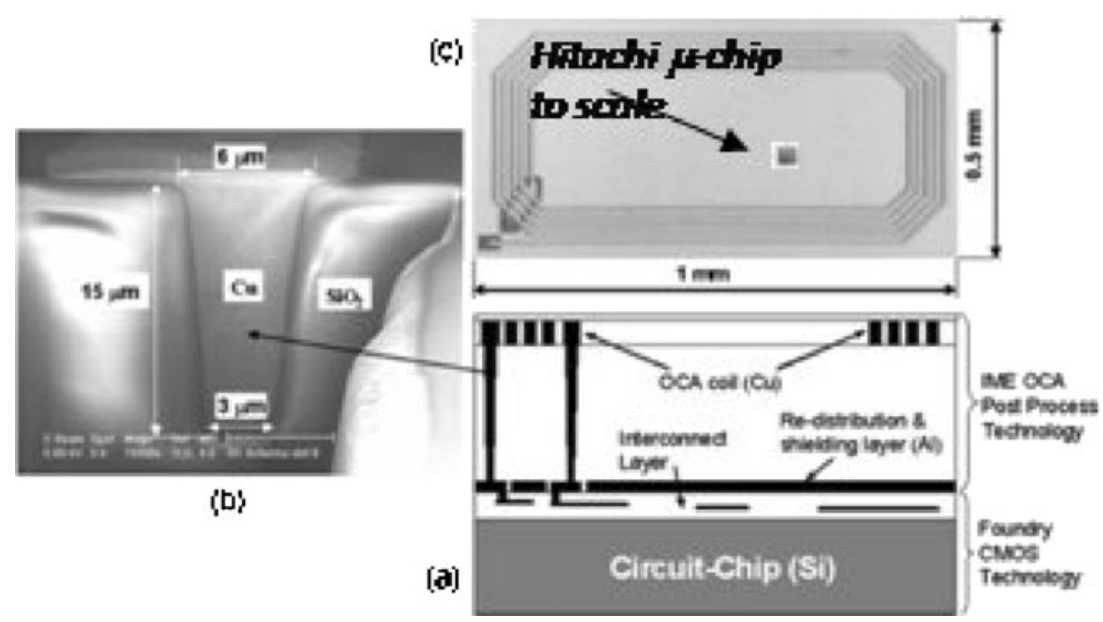

they use a heterogeneous (as opposed to single chip) approaches.

\section{Towards a nano-radio}

Our group recently demonstrated what could be called the world's smallest radio (Fig. 4 (Rutherglen and Burke 2007)), which consisted of an AM demodulator made of a single carbon nanotube (a molecular scale tube with radius of order $1 \mathrm{~nm}$ ). However, the external antenna was several $\mathrm{cm}$ in length, and the audio amplifier, speaker, and power supply (battery) were off the shelf, so the entire system volume was of order $10^{-3} \mathrm{~m}^{3}$. A similar elegant nanomechanical radio (operating in a high-vacuum environment) was also recently demonstrated by UC Berkeley (Jensen et al. 2007).

We next discuss the size of circuitry, antenna, and complete system size. The goal is to understand how the system size can be minimized, and how small it can go.

Fig. 4 Carbon nanotube radio

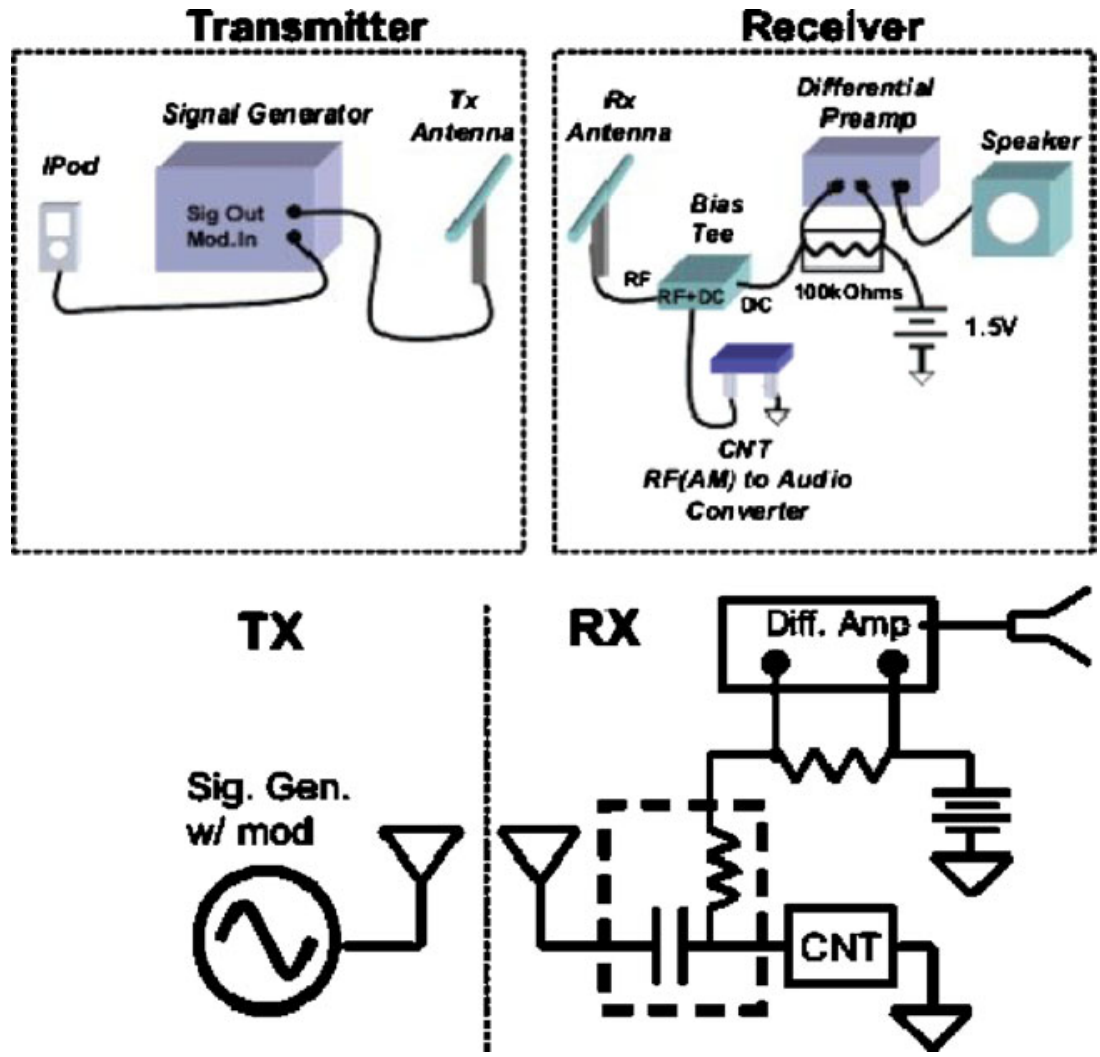




\section{Summary of small radios}

We have compiled in Table 1 below some representative sizes for the circuit, antenna, and complete radio system, from the literature (Bouvier et al. 1997; Abrial et al. 2001; Hill 2003; Usami et al. 2003; Rutherglen and Burke 2007; Usami et al. 2007). This comparison is meant to give an overview of various technical approaches (and so is not to be considered an "apples to apples" comparison), and to illustrate the state of the art and the relative importance of antenna volume in total system size. From this it is clear that the small circuit size is possible, but having a small antenna size is more challenging.

We also show in Table 1 our estimate for the size of a possible single-chip radio using "COTS" (commercial off the shelf) technology (discussed below), as well as possible advances using nanotechnology. These estimates show that a single cell radio is not completely out of reach using existing technology. In Fig. 5 below, we show the system size and single cell size of various existing and possible radio systems.

\subsection{Nano-antennas}

The field of antenna studies which are smaller than an electrical wavelength is termed electrically small antennas. We have proposed, for example, to use novel quantum properties of a single carbon nanotube to make a resonant antenna with size about $100 \times$ smaller than a classical dipole antenna for a given frequency. Such a concept is indicated schematically in Fig. 6 below(Burke et al. 2006).

While the technology to build such prototype antennas exists(Li et al. 2004; Yu et al. 2004), the predicted losses due to ohmic currents in the arms of the antenna are severe.

Table 1 Estimated circuit, antenna, and system size for various radios complied from the literature

\begin{tabular}{llll}
\hline & $\begin{array}{l}\text { Circuit size } \\
\left(\mathrm{m}^{3}\right)\end{array}$ & $\begin{array}{l}\text { Antenna size } \\
\left(\mathrm{m}^{3}\right)\end{array}$ & $\begin{array}{l}\text { System } \\
\text { size }\end{array}$ \\
\hline Hitachi & $1 \mathrm{E}-14$ & $1 \mathrm{E}-08$ & $1 \mathrm{E}-08$ \\
UCI CNT Radio & $1 \mathrm{E}-23$ & $1 \mathrm{E}-05$ & $1 \mathrm{E}-03$ \\
France-Telecom & $1 \mathrm{E}-09$ & $1 \mathrm{E}-09$ & $1 \mathrm{E}-09$ \\
Smart Dust & $3.125 \mathrm{E}-09$ & $1 \mathrm{E}-06$ & $1 \mathrm{E}-06$ \\
SMS & NA & NA & $1 \mathrm{E}-06$ \\
BioRasis & NA & NA & $5 \mathrm{E}-09$ \\
ISSYS & NA & NA & $1 \mathrm{E}-06$ \\
Potential single-chip radio & & & $1 \mathrm{E}-14$ \\
Volume of single cell & & & $1 \mathrm{E}-18$ \\
Potential nano radio & & & $1 \mathrm{E}-21$ \\
\hline
\end{tabular}

These are estimates only, as most literature does not specify complete system volume

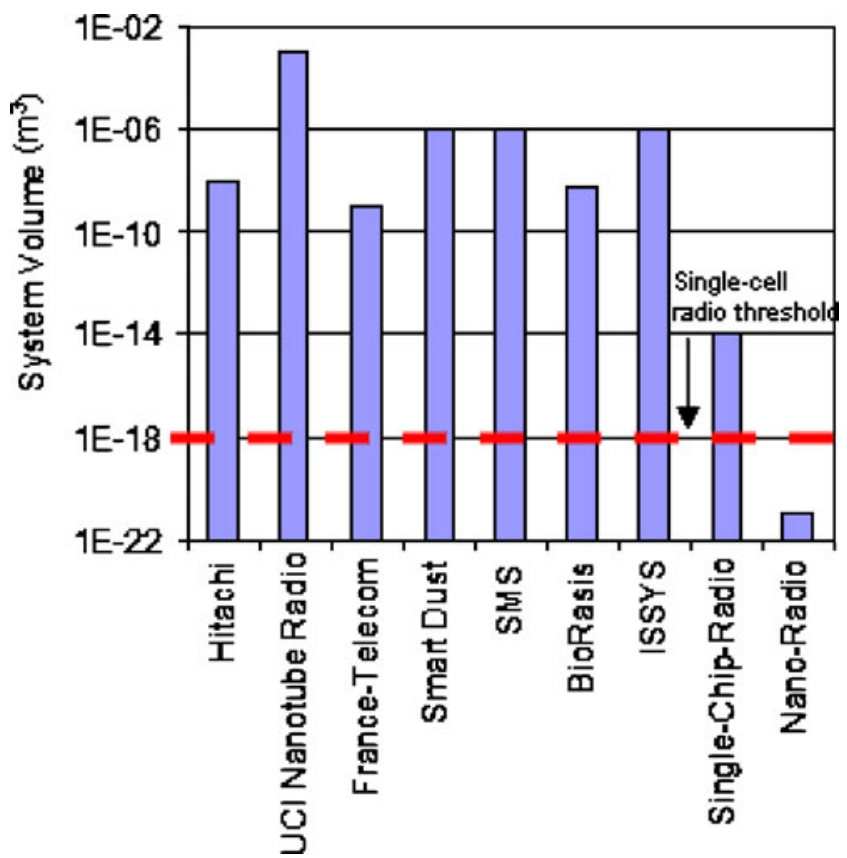

Fig. 5 Sizes of various existing and proposed radios

Our simple estimate is $-90 \mathrm{~dB}$ loss from this effect (Burke et al. 2006). More rigorous calculations are in progress along these lines. In principle, this loss can be overcome by higher intensity input radiation. However, this could result in significant heating of the antenna itself and possibly the surrounding tissue. This issue of heat, energy, voltage and current is discussed in more depth below.
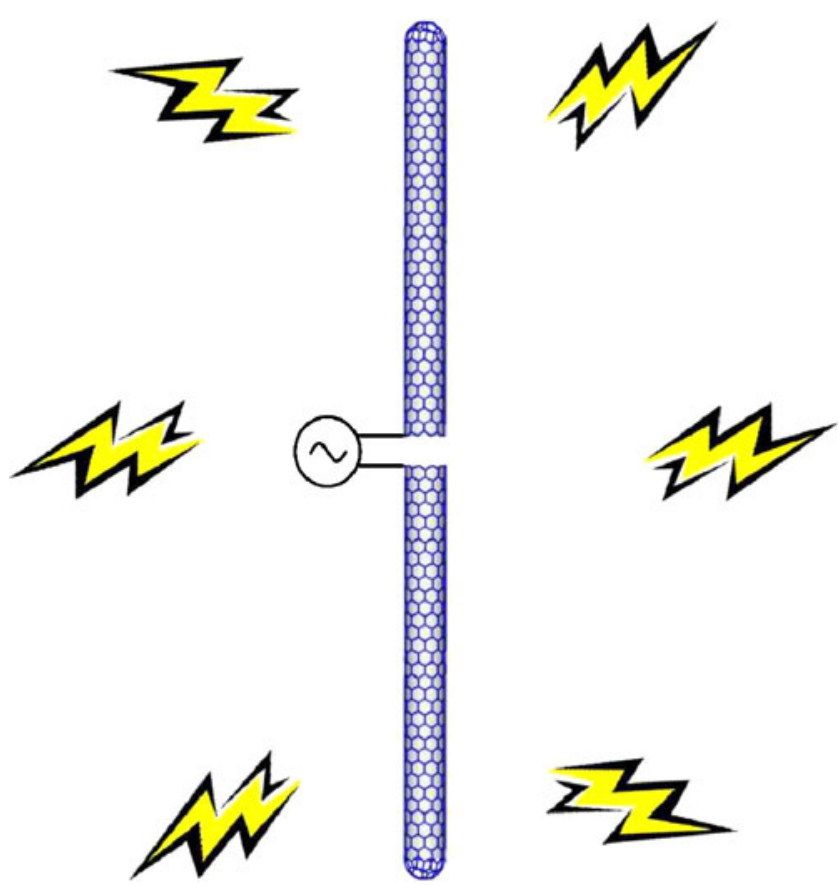

Fig. 6 Carbon nanotube antenna concept 


\subsection{Nano-antenna vs. classical antenna}

As discussed above, nano-antennas have severe energy loss problems due to the ohmic currents in the arms of the antennas. On the other hand, in classical metal antennas these ohmic currents are insignificant and generally avoided by having large enough antennas. The issue of the transition from nano-antenna to classical antenna is not yet worked out in detail, and an important topic for future study. Using existing technology it should be possible to design tiny (micron scale) low-loss metallic antennas that do not absorb much radiation, and hence can be integrated into a small, single cell radio.

\section{RF nano-heaters}

Above, we have argued ohmic losses in small antennas are a problem for signal reception and delivery to circuitry. Another approach to the absorption of RF power is to use it as a local heater, which can be used to effect biochemistry at the nanoscale for nanotechnology investigations and potential therapeutic applications. This is another form of "RF remote control" of biological function, which uses heat rather than circuitry to control chemistry. While there are many possibilities, we summarize two examples from the literature using various forms of RF Nano-heaters: One targeted towards therapeutics, and the other a more basic proof of principle demonstration.

\subsection{Therapeutic heaters}

When heated above a certain temperatures, tissues undergo necrosis; this effect is called thermoablation. The fundamental hypothesis of thermoablative cancer therapy currently under study is that RF absorbing nanoparticles can be selectively localized to cancer tissue (using, e.g., antibodies to target specific tissues), allowing heat to be applied locally with minimal or no damage to surrounding, healthy tissue. Both nanoparticles and nanotube antennas have been proposed for this purpose.

Iron oxide nanoparticles can be used to absorb RF or AC magnetic field energy through eddy currents. Several proof of principles studies have been published(Ito et al. 2004, 2007; DeNardo et al. 2005; Ivkov et al. 2005; Sonvico et al. 2005; Wust et al. 2006; Majewski and Thierry 2007). Carbon nanotubes can also serve as nano-antenna absorbers to heat locally tissue. However, the specific absorption of the material is not well-understood at the current state of the $\operatorname{art}(G a n n o n$ et al. 2007). In all cases, in addition to better understanding of the RF nano-antenna interaction, the most significant issue to be resolved are the pharmico-kinetics and localization of the absorbers with sufficient specificity to allow targeted application of heat for therapeutic effects with little or no damage to surrounding tissue.

5.2 RF remote control: turning heat to biochemical activity

DNA hybridization/dehybridization is well know as a temperature sensitive event. In (Hamad-Schifferli et al. 2002), Au nanoparticles (NP) were covalently linked to DNA, and exposed to a $1 \mathrm{GHz}$ RF field. This caused heating of the Au NPs, and reversible dehybridization. This is clear demonstration of RF remote control of molecular scale biochemistry. Further development of such techniques, similar to therapeutic techniques discussed above, will require close attention to local heating at the nanoscale, clearly addressing global RF absorption of the surrounding environment.

\section{Heater vs. radio: what is information?}

For a truly nanoscale radio, the concepts of integrated electronic memory, signal processing, and currents and voltages may need to be revised. The two-way flow of information as depicted schematically in Fig. 1 above is a traditional way of thinking about information flow in engineering systems.

Is it possible to have remote control of cell operation using RF in vivo, with different concepts from the current/ voltage used in electrical RF engineering? Some ideas deal with heating, but this is just a step. We have only scratched the surface. We have fundamental concepts of heat for nanodetectors, and current/voltage for macro/microcircuits. The Au NP RF absorption (Hamad-Schifferli et al. 2002) is a translation of heat to a biomolecular event. However, signal transduction in cells occurs by completely different "circuit elements", by phosphorylization and de-phosphorylization of proteins by kinases and concomitant enzyme allosteric conformational activity, and the resultant binding/ unbinding of DNA expression inhibitors/promoters, and the subsequent concentration of proteins expressed by various genes. Also, small molecule concentration ratios (e.g. ATP to ADP) provided metabolic information. How to translate between these two information carrying worlds is a question that should be addressed by the academic community, and may hold promise for many diverse biomedical applications yet to be dreamed of.

\subsection{Fundamental limits}

In some sense, the world of electrical engineering is incompatible with the signal processing hardware prevalent in biological systems. So, scaling down the entire "top down" RFID circuit project may be doomed to failure from 
the get go. In any case, it should be interesting to determine what the limits are on the development of traditional RF engineering and single cell RFID. Ultimately, there is a fundamental relationship between energy, entropy, heat, and information, and we conjecture this will set a fundamental limit on the interaction between electronics and biological systems at the single cell and biochemical level.

\section{Prospects for the future}

Above we have discussed the state of the art RFID technologies as well as some general issues regarding scaling these technologies down to the single cell limit. We next discuss possible new and future research directions towards the goal of integrated nano-radio systems. We propose three broad directions, in increasing levels of ambition, challenge, and significance.

7.1 Step 1: a vision for a unified, single-chip universal platform

A common, uniform, and standardized interface between a single-chip radio and the outside world would be very useful for the research community. Our proposed goal is indicated schematically in Fig. 7 below, and would achieve the following milestones:

- $100 \times 100 \times 5 \mu \mathrm{m}$ CMOS radio chip

- On-chip-antenna

- No battery required

- Two-way communication enabled

- Real-estate for custom sensors (e.g. nanowire sensors, electrochemical sensors, nanotube sensors, etc.)

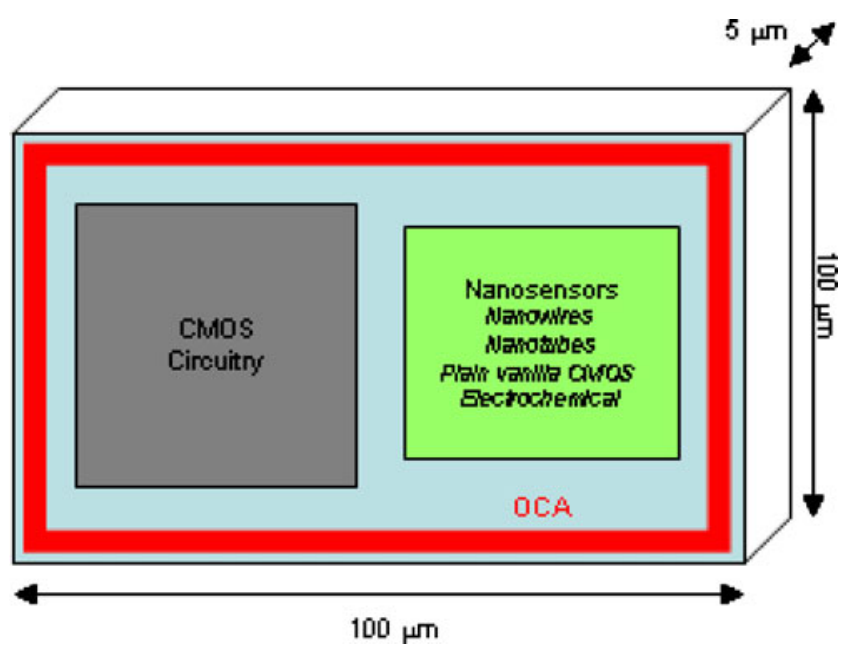

Fig. 7 Micro-radio concept

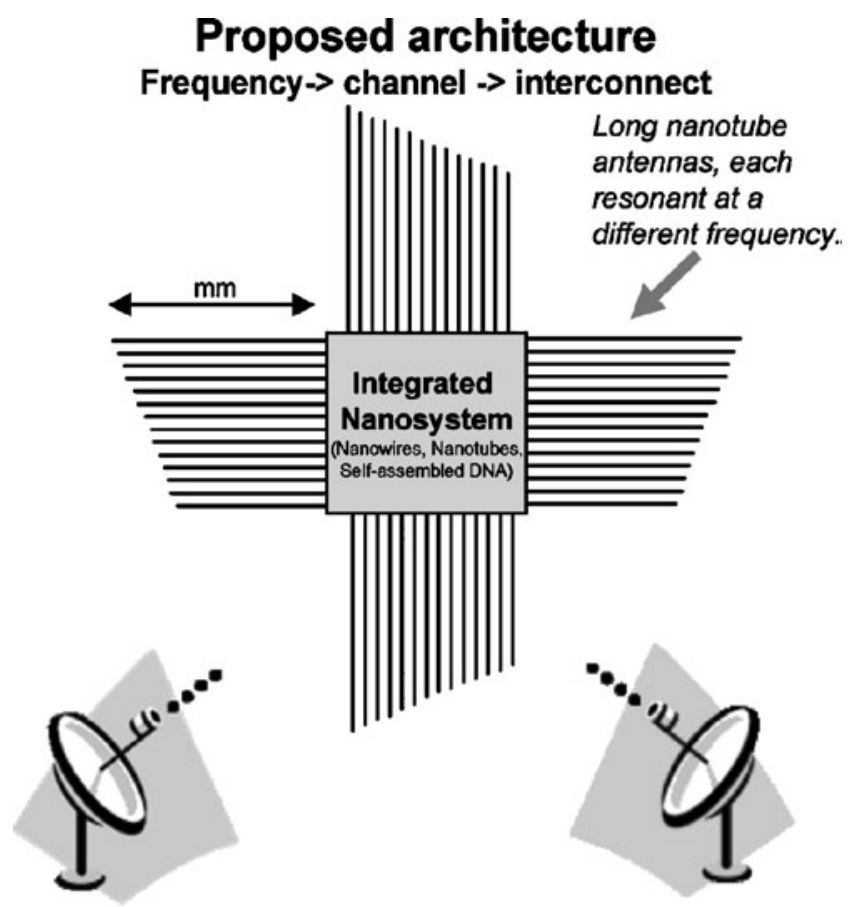

Fig. 8 Possible architecture for integrated nanosystems

The development of such a "platform" would enable the research community to integrate (post fabrication) various sensors onto the chip. As a first application, glucose sensors for diabetes monitoring would be a good target. However, in general there is a myriad of possible biological events to be monitored in vivo, and new sensing technologies are constantly emerging. A single RF platform to interface to these new technologies would be a significantly accelerate the application of new sensing and nanotechnologies in the life sciences and biomedical device field.

\subsection{Challenges}

The first challenge regards the antenna, and the amount of RF power required to couple if the antenna is electrically small. For a tiny radio to work, would RF fields need to be so high as to damage surrounding tissue? Are ultra-high frequency (e.g. $60 \mathrm{GHz}$ ) signals needed for small enough antennas, and how is this going to propagate through tissue? The on-chip-antennas developed above are already sub-mm in length, so the prospect of a $0.1 \times 0.1 \mathrm{~mm}$ antenna seems feasible short term. The goal of integrating this onto a CMOS chip is also feasible, since it has been demonstrated in other applications as well.

\subsection{Step 2: development of a single cell radio?}

Once step 1 is achieved, the next goal will be to determine the fundamental scaling limit of such a tiny RFID chip 
technology. Key challenges will be the miniaturization of the antenna, and getting enough power coupled through smaller and smaller antennas. Miniaturization of the circuitry itself may also become an issue as the circuitry will need to fit into an area comparable to one square micron. This would enable roughly 1000 components in the $30 \mathrm{~nm}$ node, so resource starved circuitry and computing would need to be seriously addressed.

These research challenges are difficult engineering issues, but all of them seem fundamentally solvable. In particular, new fabrication technologies are not needed or assumed in the development of a single cell size radio. However, to get a radio that will fit into a cell easily, nanotechnology will be needed, as is discussed next.

\subsection{Step 3: integrated nanosystems}

The long term goal of a sub-micron sized nano-robot with computing and communications ability is the realm of nanotechnology, and currently does not exist. This goal can be best stated as a vision, because the particular roadmap to get there (and even the best route) cannot be clearly laid out given our current state of knowledge of how RF fields interact with nanosystems. We have proposed one possible implementation of such a vision, using nanotube antennas and frequency domain multiplexing for high-bandwidth communication with integrated nanosystems (Fig. 8). One of the main challenges (in addition to the RF interface) is the development of integrated nano-circuits, which also is predicated on economical, precision nano-fabrication. The latter is a grand challenge beyond the scope of this paper, but being addressed by the research community at large via self-assembly, DNA controlled assembly, etc. The vision of a nano-radio is thus to be considered one (important) application of a yet to be developed nanotechnology.

\section{Conclusions}

The key components to reducing radio size are the antenna and the battery. Using the RF field to generate on-chip power completely obviates the need for the battery. Using an on-chip antenna allows for smaller system sizes. Even with an on-chip antenna, the system sizes demonstrated to date and immediately feasible are dominated by the antenna size, not the circuitry. In order to address this issue, we have proposed nano-antennas. More research is needed to address the trade-offs between efficiency, required external power, antenna size, and heating. A single chip (including antenna) radio system (with room for on-board sensors) of size $100 \times 100 \mu \mathrm{m}$ by $1 \mu \mathrm{m}$ seems feasible with current technology. It remains an open question whether such an approach can be taken to develop a single-cell radio system.
A true nano-radio will require further developments in nano-fabrication technology, an issue which is currently being addressed at a world-wide level.

Open Access This article is distributed under the terms of the Creative Commons Attribution Noncommercial License which permits any noncommercial use, distribution, and reproduction in any medium, provided the original author(s) and source are credited.

\section{References}

A. Abrial, J. Bouvier et al., A new Contactless Smart Card IC using an on-chip antenna and an asynchronous microcontroller IEEE J. Solid-State Circuits 36(7), 1101-1107 (2001)

S. Ahson, M. Ilyas, RFID handbook: applications, technology, security, and privacy (Taylor \& Francis, Boca Raton, 2008)

J. Bouvier, Y. Thorigne, et al., A smart card CMOS circuit with magnetic power and communication interface. Solid-State Circuits Conference, 1997. Digest of Technical Papers. 44th ISSCC., 1997 IEEE International (1997)

P. Burke, Z. Yu et al., Quantitative theory of nanowire and nanotube antenna performance IEEE Trans. Nanotechnol. 5(4), 314-334 (2006)

S.J. DeNardo, G.L. DeNardo et al., Development of tumor targeting bioprobes (111In-chimeric L6 monoclonal antibody nanoparticles) for alternating magnetic field cancer therapy Clin. Cancer Res. 11(19), 7087s-s7092 (2005)

K. Finkenzeller, RFID handbook: fundamentals and applications in contactless smart cards and identification (Wiley, Hoboken, NJ, 2003)

C.J. Gannon, P. Cherukuri et al., Carbon nanotube-enhanced thermal destruction of cancer cells in a noninvasive radiofrequency field Cancer 110(12), 2654-2665 (2007)

L.H. Guo, A.P. Popov et al., A small OCA on a $1 \times 0.5-\mathrm{mm}^{2} 2.45-\mathrm{GHz}$ RFID tag - design and integration based on a CMOS-compatible manufacturing technology IEEE Electron Device Lett. 27(2), 9698 (2006)

K. Hamad-Schifferli, J.J. Schwartz et al., Remote electronic control of DNA hybridization through inductive coupling to an attached metal nanocrystal antenna Nature 415(6868), 152-155 (2002)

J.L. Hill, System architecture for wireless sensor networks. (Berkeley, CA, University of California, Berkeley, 2003). Ph.D.: 166

A. Ito, Y. Kuga et al., Magnetite nanoparticle-loaded anti-HER2 immunoliposomes for combination of antibody therapy with hyperthermia Cancer Lett. 212(2), 167-175 (2004)

A. Ito, M. Fujioka et al., 4-S-Cysteaminylphenol-loaded magnetite cationic liposomes for combination therapy of hyperthermia with chemotherapy against malignant melanoma Cancer Sci. 98(3), 424-430 (2007)

R. Ivkov, S.J. DeNardo et al., Application of high amplitude alternating magnetic fields for heat induction of nanoparticies localized in cancer Clin. Cancer Res. 11(19), 7093s-7103s (2005)

K. Jensen, J. Weldon et al., Nanotube radio Nano Lett. 7(11), 3508 $3511(2007)$

K. O, K. Kim et al., On-chip antennas in silicon ICs and their application IEEE Trans. Electron Dev. 52(7), 1312-1323 (2005)

$\mathrm{S}$. Li, Z. Yu et al., Electrical properties of $0.4 \mathrm{~cm}$ long single walled carbon nanotubes Nano Lett. 4(10), 2003-2007 (2004)

P. Majewski, M. Thierry, Functionalized magnetite nanoparticlessynthesis, properties, and bio-applications Crit. Rev. Solid State Mater. Sci. 32(3), 203-215 (2007) 
C. Rutherglen, P. Burke, Carbon nanotube radio Nano Lett. 7(11), 3296-3299 (2007)

A. Shamin, L. Roy, et al., On-chip antenna measurements: calibration and de-embedding considerations. IMTC 2005-Instrumentation and Measurement Technology Conference. Ottawa, Canada, pp 463-466, 2005

A. Shamin, L. Roy et al., $24 \mathrm{GHz}$ On-chip antennas and balun on bulk Si for air transmission IEEE Trans. Antennas Propag. 56(2), 303$311(2008)$

F. Sonvico, S. Mornet et al., Folate-conjugated iron oxide nanoparticles for solid tumor targeting as potential specific magnetic hyperthermia mediators: synthesis, physicochemical characterization, and in vitro experiments Bioconjugate Chem. 16(5), $1181-1188(2005)$
M. Usami, A. Sato, et al., Powder LSI: an ultra small RF identification chip for individual recognition applications. Solid-State Circuits Conference, 2003. Digest of Technical Papers. ISSCC. 2003 IEEE International (2003)

M. Usami, H. Tanabe, et al., A $0.05 \times 0.05 \mathrm{~mm}^{2}$ RFID Chip with Easily Scaled-Down ID-Memory. Solid-State Circuits Conference, 2007. ISSCC 2007. Digest of Technical Papers. IEEE International (2007)

P. Wust, U. Gneveckow et al., Magnetic nanoparticles for interstitial thermotherapy - feasibility, tolerance and achieved temperatures Int. J. Hypotherm. 22(8), 673-685 (2006)

Z. Yu, S. Li et al., Synthesis of aligned arrays of millimeter long, straight single walled carbon nanotubes Chem. Mater. 16(18), 3414-3416 (2004) 